\title{
Wearable Augmented Reality Displays
}

\author{
Shoaib R. Soomro, Erdem Ulusoy, Hakan Urey \\ Koc University, Electrical Engineering Department, Optical Microsystems Laboratory, Istanbul, Turkey \\ *hurey@ku.edu.tr
}

\begin{abstract}
Wearable augmented reality displays and associated light efficient screens are presented. We review three recent developments in augmented reality displays: head-mounted projection display, retro-reflective augmented reality screen and light efficient 3D augmented reality display.

OCIS codes: (120.2040) Displays; (120.2820) Heads-up displays; (240.0240) Optics at surfaces.
\end{abstract}

\section{Introduction}

Compact, comfortable and low power 2D/3D displays are widely investigated for augmented reality applications. The general problems with wearable augmented reality displays (particularly near-to-eye displays) are the restricted field of view, low light efficiency and involvement of bulky optical elements. In this paper, as an alternative to near-to-eye displays, we present the wide field of view, compact and light-efficient displays based on head worn pico-projectors andretro-reflective screensthat have been recently developed in our group for $2 \mathrm{D} / 3 \mathrm{D}$ augmented reality applications [1-7].
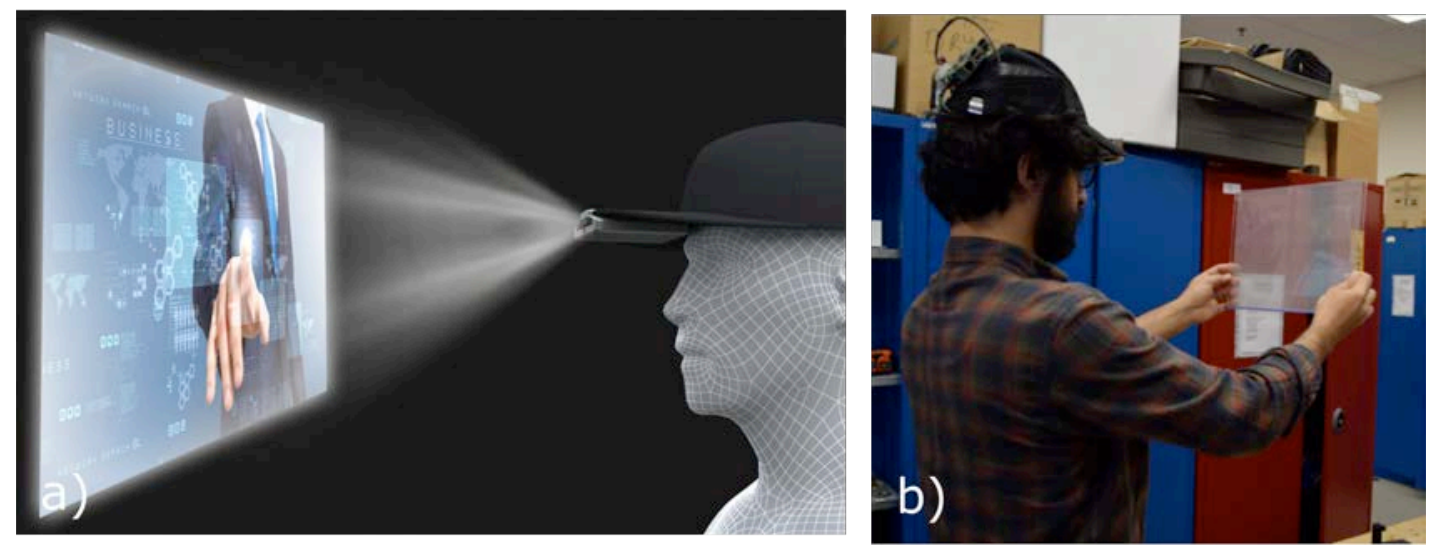

Figure 1: (a) Conceptual illustration of head-mounted projection display together with an arbitrary projection surface as screen, and (b) Shows the HMPD prototype used with transparent retro-reflective screen.

First, we introduce a compact head-worn display assembled around a regular cap. The system includes a MEMS projector based standalone wearable display providing the required computation, connectivity and power on the head and is used together with retro-reflective surfaces as image transfer screens [1-3]. The light from the wearable projector is directed back with minimum scattering and provides the viewer with bright displayed content for wide range of distance. Second, we present a highly transparent retro-reflective screen for augmented reality applications. The screen is based on retro-reflective microspheres partially patterned on optically clear surface. The retroreflective feature of screen provides light-efficiency for content display, while the partial patterning facilitates the higher optical transparency. Finally, we introduce a light efficient and wide field of view 3D augmented reality display using a projector pair and see-through retro-reflective screens. The display delivers stereo $3 \mathrm{D}$ content to viewer by allocating one projector per eye, while maintaining real-world view on the other side of screen.

\section{Head Mounted Projector}

The prototype is a standalone pico-projection based wearable augmented reality display for overlaying digital content on top of real objects. The display has form factor of a standard cap as shown in fig. 1a. The display consists of a 30-lumen laser sourced pico-projector in front providing the HD (720p) resolution. The projector illuminates the screen (preferably a retro-reflective fabric) to augment virtual content on to the scene. The identical perspective of projector and viewer with respect screen provides undistorted content to viewer even when the projection surface is highly rough (i.e. corner of a room, wrinkled cloth). 


\section{Retro-reflective Augmented Reality Screens}

We present a novel screen that utilizes the retro-reflective materials to produce a highly transparent and lightefficient screen [4]. A regular diffused screen scatters incoming light into the full hemisphere, while a retroreflective surface directs major portion of incoming light back towards the projector. The narrow scattering of light around the projector provides bright content to the eyes positioned close to projector. The optical transparency is added by using an optically clear surface (i.e glass) as screen substrate, which is partially patterned with the retroreflective microspheres. Through partial patterning, the total screen area is divided into retro-reflective and transmissive regions $[4,5]$.
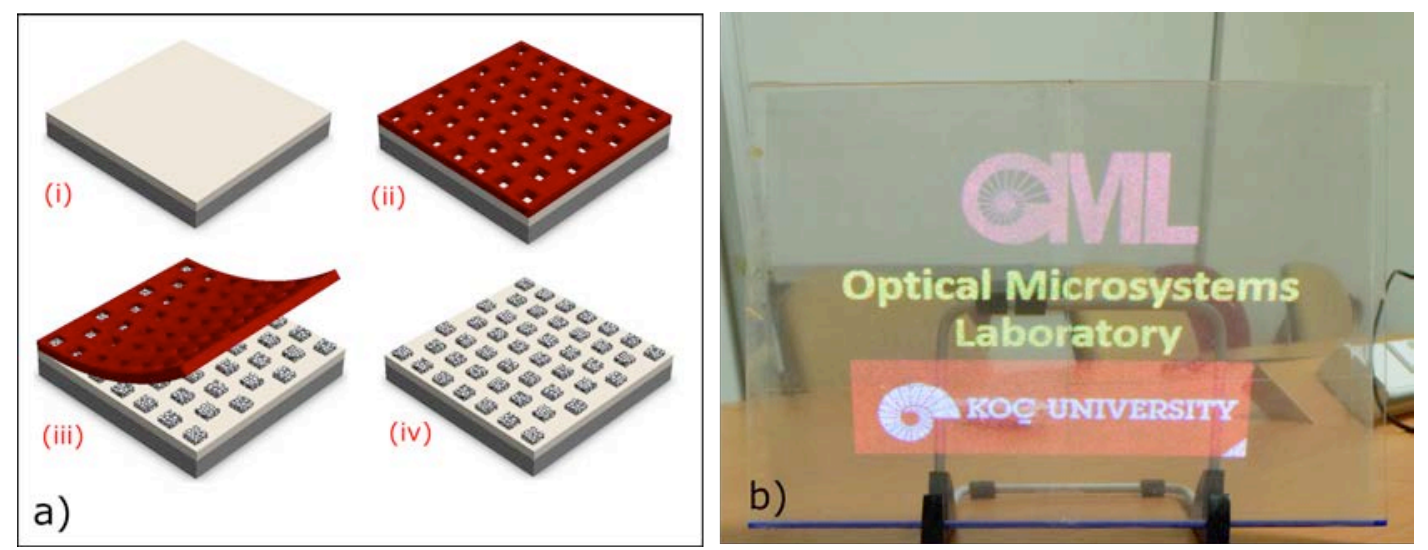

Figure 2:(a) Fabrication process of transparent retro-reflective screen, involving a thin adhesive coating on clear substrate (i), stencil mask bonding (ii), microsphere spread and mask removal (iii), b) Shows the content displayed on transparent screen through a projector and viewed from eye position [4].

To minimize the visibility of structural artifacts and utilize full resolution of projector, the microspheres are patterned as tiny square shaped islands irresolvable to the viewer at the working distance. Fig. 2(a) illustrates the fabrication recipe of the screen, where a clear substrate is prepared and coated with a thin layer of optical adhesive. After that, a large size hard but thin mask (steel stencil) having the negatively patterned perforations is deposited to surface. The microspheres are then spread across and the mask perforations are filled with microspheres. The mask is gently removed after the microspheres are submerged inside the adhesive. We have fabricated the screens providing a transparency of $50 \%$ to $90 \%$. Our results, showed that the screen can provide several times more brightness than a non-transparent diffused screen at closer distances $(40-70 \mathrm{~cm})$ and up to 100 times more brightness at farther distances $(300 \mathrm{~cm})$. Fig. 2(b) shows the content on the screen as seen by a viewer wearing the head mounted projector.

\section{Light Efficient 3D Augmented Reality Displays}

We present a light efficient 3D augmented reality display using highly transparent desktop size retro-reflective screen as shown in Fig. 3(a). The display provides bright stereoscopic content on a transparent retro-reflective screen. The display uses a pair of laser sourced pico-projector positioned close to viewer's eyes, where each projector illuminates the screen with a single view of stereoscopic content [6]. Due to the retro-reflective nature of the screen, the respective projector light is reflected back and the eye positioned close to projector perceives very bright content displayed on screen. The interpupillary distance between the projectors separates the stereoscopic content for each eye. The highly transparent retro-reflective screen also facilitates the viewer to clearly see the real world scene on the other side of the screen. Our screen is the large size version of transparent retro-reflective screen described in previous section. 

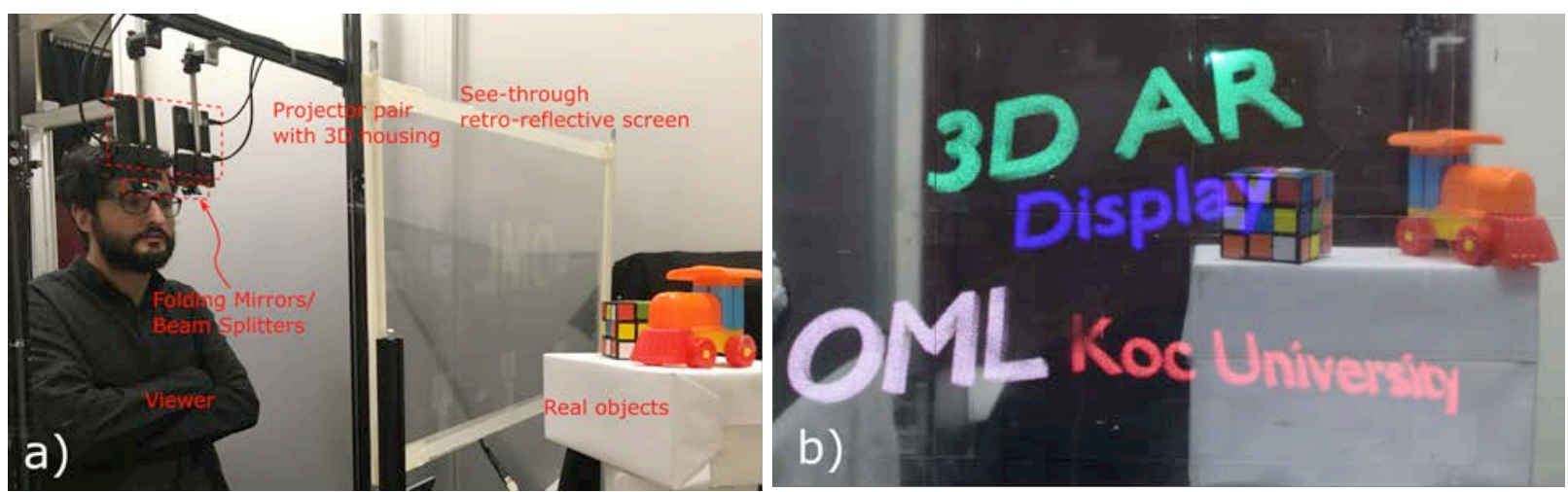

Figure 3: (a) Experimental prototype of 3D augmented reality display [6] and, (b) The content displayed through one of the two projectors.

We developed the prototype display using 30 lumens low power laser sourced projectors and $60 \times 40 \mathrm{~cm}^{2}$ retroreflective screen providing $75 \%$ transparency. The display was tested for various viewing conditions, including bare eye and beam-splitters based settings. The presented display can provide $300-1000 \mathrm{~cd} / \mathrm{m}^{2}$ brightness per eye for a working distance of up to $200 \mathrm{~cm}$. The stereo crosstalk of the system was measured to be $<5 \%$.

\section{References}

[1] K. Akşit, D. Kade, O. Özcan, and H. Urey. "Head-worn mixed reality projection display application," In Proceedings of the 11th Conference on Advances in Computer Entertainment Technology, pp. 11:1-11:9 (2014).

[2] D. Kade, K. Akşit, H. Urey, and O. Özcan, "Head-mounted mixed reality projection display for games production and entertainment," Personal and Ubiquitous Computing, 19(3), 509-521 (2015).

[3] Ç. Genç, S. Soomro, Y. Duyan, S. Ölçer, F. Balcı, H. Ürey, and O. Özcan. "Head Mounted Projection Display \& Visual Attention: Visual attentional processing of head referenced static and dynamic displays while in motion and standing," In Proceedings of the ACM CHI Conference on Human Factors in Computing Systems, pp. 1538-1547 (2016).

[4] S.R Soomro, and H. Urey, "Design, fabrication and characterization of transparent retro-reflective screen." Opt. Exp. 24(21), 24232-24241 (2016).

[5] S.R. Soomro, and H. Urey, "Retro-reflective Characteristics of Transparent Screen for Head Mounted Projection Displays." OSA Frontiers in Optics, FTu5A-2. (2016).

[6] S.R. Soomro, and H. Urey, "Augmented reality 3D display using head-mounted projectors and transparent retro-reflective screen". In Proceedings of SPIE 101260 (2017).

[7] H. Urey, et al. "MEMS scanners and emerging 3D and interactive augmented reality display applications." In proceedings of 17th IEEE International Conference on Transducers \& Eurosensors, pp. 2485-2488 (2013). 